\title{
Inhaltsanalytische Modelle zur Identifikation und Analyse von ethischen Rechtfertigungen politischer Gewalt
}

\author{
Susanne Halverscheid/Erich H. Witte*
}

\begin{abstract}
The purpose of this article is to present two theoretical frameworks that have been useful in identifying and analyzing ethical justifications for politically motivated acts of violence. The first concept is based on Kienpointner's model of argumentation patterns ${ }^{1}$ and aims at extracting justifications based upon argumentation analysis. The underlying ethical principles can then be examined by employing the prescriptive attribution model $^{2}$ that differentiates between twelve ethical positions. The analytical frameworks will be presented and exemplified by findings obtained in the analysis of justifications of war and terror.
\end{abstract}

Keywords: Rechtfertigung, Empirische Ethikforschung, Moral, Krieg, Terror

\section{Politische Gewalt und Moral}

$\mathrm{S}$ eit mehr als 2000 Jahren haben Philosophen sich der Frage gewidmet, unter welchen Bedingungen Kriege und andere Formen der Gewalt moralisch vertretbar sind. Die Erarbeitung von Kriterien, anhand derer geprüft werden sollte, ob in einem konkreten Fall ein rechtfertigender oder gar zwingender Grund vorliege, eine Gewaltanwendung zu erlauben und entsprechend moralisch zu begründen, führte zur »Lehre vom gerechten Krieg « (bellum iustum) (Augustinus von Hippo [354-430], Thomas von Aquin [1225-1274]). Diese beinhaltet sowohl Kriterien für das Beginnen eines Krieges (ius ad bellum) als auch für die Kriegsführung an sich (ius in bello). Als notwendige Bedingungen für einen Kriegsbeginn gelten unter anderem ein gerechter Grund (causa iusta), die Gewaltanwendung als letztes Mittel (ultima ratio) sowie die rechte Absicht, das »Gute zu mehren oder das Böse zu meiden $\aleph^{3}$ (intentio recta). Als Maßstab für die gerechte Kriegsführung werden insbesondere die Verhältnismäßigkeit der Mittel (debitus modus) und die Unterscheidung von Kombattanten und Nichtkombattanten gefordert. Letztere Unterscheidung, die im Wesentlichen zwischen »legitimen « und »nicht-legitimen « Zielpersonen zu differenzieren versucht, wurde in jüngster Zeit auch zur Verurteilung terroristischer Anschläge herangezogen: »there is nothing here that can morally justify the most violent operations staged by Al-Qaida (...). Primarily, there is, in terms of just war theory, simply no just cause. «4

\footnotetext{
* Prof. Dr. Erich H. Witte ist Leiter des Lehrstuhls Sozialpsychologie an der Universität Hamburg. Susanne Halverscheid ist Diplom-Psychologin und widmet sich Themen der Politischen Psychologie, wie insbesondere der Konfliktbearbeitung und der ethischen Argumentation innerhalb politischer Debatten.

1 M. Kienpointner (1992). Alltagslogik. Struktur und Funktion von Argumentationsmustern. Stuttgart - Bad Cannstatt: frommann-holzboog.

2 Das präskriptive Attributionsmodell wurde von Witte \& Doll (1995) aufgestellt und später von Witte \& Halverscheid (2006) weiterentwickelt. Vgl. E. H. Witte \& J. Doll (1995). Soziale Kognition und empirische Ethikforschung zur Rechtfertigung von Handlungen. In: E. H. Witte (Hrsg.). Soziale Kognition und empirische Ethikforschung (S. 97-115). Lengerich: Pabst Science Publishers und E. H. Witte \& S. Halverscheid (2006). Justification of War and Terrorism. A comparative case study examining ethical positions based on Prescriptive Attribution Theory. (Hamburger Forschungsbericht zur Sozialpsychologie Nr. 70). Hamburg: Universität Hamburg, Arbeitsbereich Sozialpsychologie.

3 T. v. Aquin (1959). Summa theologica. Deutsche Thomas-Ausgabe, Bd. 17. Heidelberg: Kerle.

4 Vgl. S. Smilansky (2004). Terrorism, Justification, and Illusion. Ethics (114), S. 8790-8805 und C. A. J. Coady (2004). Terrorism, Morality, and Supreme Emergency. Ethics (114), S. 772-789.
}

Obgleich die Kriterien des gerechten Krieges bis heute in Diskussionen über die Legitimation politischer Gewalt angeführt werden, hat die Lehre vom gerechten Krieg mit der Einführung des Völkerrechts an Einfluss verloren. Die Schwierigkeit, klare Kriterien aufzustellen, die Gewaltanwendungen in Ausnahmefällen erlauben, ist jedoch nach wie vor aktuell. So scheinen westliche Kriege in jüngster Zeit häufiger auf der Grundlage »Humanitärer Intervention « gerechtfertigt zu werden, obgleich dieses Konzept in der Charta der Vereinten Nationen nicht verankert ist. ${ }^{5}$ Auf islamischer Seite sind dem Koran und der Sunna Regeln für den klassischen Jihad zu entnehmen. Als grundlegend wird häufig folgender koranischer Vers angeführt: „Und kämpft um Gottes Willen gegen diejenigen, die gegen Euch kämpfen! Aber begeht keine Übertretung (indem ihr den Kampf auf unrechtmäßige Weise führt)! Gott liebt die nicht, die Übertretungen begehen ${ }^{6}{ }^{6}$ So werden auch hier konkrete Prinzipien aufgeführt, die die Verhältnismäßigkeit der Mittel und das Verhalten gegenüber Kriegsgefangenen und der Zivilbevölkerung festlegen. ${ }^{7}$

Im Kontext der Thematik vom »11. September«, dem »jihad gegen Ungläubige« und dem »Krieg gegen den Terror« sind zahlreiche Veröffentlichungen erschienen, die aus moralischer Sicht zu klären versuchen, wann ein Akt der Gewalt vertretbar sein könnte. Bislang liegen jedoch kaum Arbeiten vor, in denen die grundlegenden ethischen Rechtfertigungsmuster kriegerischer und terroristischer Akte systematisch untersucht wurden. Von einem Versuch, diese ethischen Prinzipien im Rahmen einer vergleichenden Fallstudie gegenüberzustellen, soll an dieser Stelle berichtet werden. Theoretischer Hintergrund ist dabei die empirische Ethikforschung - ein Gebiet, das sich inhaltlich auf Ethik-Ansätze der praktischen Philosophie stützt, zugleich aber empirische Analysemethoden zur Auswertung heranzieht.

\footnotetext{
5 W. Ruf (1994). Die neue Welt-UN-Ordnung. Münster: Agenda.

6 Sure 2:190, zitiert nach R. Paret (2001). Der Koran. Übersetzung von Rudi Paret (8. Aufl.). Stuttgart: Kohlhammer.

7 Vgl. B. Tibi (2002). War and Peace in Islam. In: S. H. Hashmi (Hrsg.). Islamic Political Ethics. Civil Society, Pluralism, and Conflict (S. 175-193). Princeton: Princeton University Press.
} 


\section{Begriffsbestimmung: Moral und Ethik}

Obwohl »Ethik« und »Moral« von ihrer Wortgeschichte mehr oder weniger gleichbedeutend sind (das griechische »ethos « und das lateinische »mos« bedeuten jeweils soviel wie Sitte und Gewohnheit), stehen »Ethik« und »Moral« in der praktischen Philosophie für Zweierlei: Der Begriff der Moral bezeichnet ein komplexes und vielschichtiges System von konkreten Regeln, Normen und Wertmaßstäben. Ethik hingegen ist eine philosophische Disziplin, »die sich mit den verschiedenen Erscheinungsformen der Moral und den mit ihnen zusammenhängenden Theoriefragen beschäftigt. $\ll^{8}$ Ethik kann demnach auch als »Moralphilosophie « bezeichnet werden. »Sie operiert gegenüber der Ebene der Moral auf einer Metaebene und verhält sich zur Moral ähnlich wie die Rechtsphilosophie zum Recht oder die Religionsphilosophie zur Religion. «9

\section{Empirische Ethikforschung}

Zentraler Gegenstand der empirischen Ethikforschung ist die Frage nach der Angemessenheit einer Handlung. Wie rechtfertigen Personen strittige Handlungen? Wie werden verschiedene Handlungsalternativen ethisch bewertet? Welche ethischen Grundpositionen werden in den Rechtfertigungen deutlich? ${ }^{10}$ Rechtfertigen wird dabei definiert als das Erheben des Anspruchs auf eine "nicht-negative oder positive Bewertung eines Sachverhalts, für dessen Zustandekommen ein Subjekt verantwortlich ist « ${ }^{11}$. Rechtfertigungen werden gegeben, wenn durch das eigene Handeln moralische Normen verletzt worden sind. Dabei gilt es, Gründe für die Handlung vorzubringen, die ein Gegengewicht zur begangenen Werteverletzung darstellen. Im Gegensatz zur Entschuldigung wird die Verantwortlichkeit für die umstrittene Handlung zugestanden, die zugeschriebene negative Eigenschaft der Tat jedoch bestritten. ${ }^{12}$

Rechtfertigungen unterscheiden sich bezüglich der ethischen Prinzipien, mittels derer eine Handlung als ‘gut ‘ oder sschlecht beurteilt wird. Dieser Zuschreibungsprozess ist Gegenstand der empirischen Ethikforschung und wird als präskriptive Attribution bezeichnet. Im Gegensatz zur deskriptiven Attribution, in der Sachverhalte durch die Angabe von Ursachen und Gründen erklärt werden, bewegen sich präskriptive Attributionen auf der Werteebene.

Die empirische Untersuchung ethischer Grundpositionen erfolgt in einem Zweischritt. Zunächst gilt es, aus relevantem Material politischer Äußerungen diejenigen Aussagen zu selektieren, die eine Rechtfertigung darstellen (vgl. 3.1). Anschlie-

8 D. Birnbacher (2003). Analytische Einführung in die Ethik (S. 429). Berlin: Walter de Gruyter.

9 Ebenda, S. 2.

10 Vgl. E. H. Witte (2001). Ethische Grundpositionen und ihre Bedeutung bei der Rechtfertigung beruflicher Handlungen. (Hamburger Forschungsbericht zur Sozialpsychologie Nr. 38). Hamburg: Universität Hamburg, Arbeitsbereich Sozialpsychologie.

11 Klein, J. (1987). Die konklusiven Sprechhandlungen. Studien zur Pragmatik, Semantik, Syntax und Lexik von Begründen, Erklären-warum, Folgern und Rechtfertigen (S. 241). Tübingen: Niemeyer.

12 J. Rehbein (1972). Entschuldigungen und Rechtfertigungen. Zur Sequenzierung von kommunikativen Handlungen. In: D. Wunderlich (Hrsg.). Linguistische Pragmatik (S. 288-317). Frankfurt a. M.: Athenäum. ßend werden die identifizierten Rechtfertigungen bezüglich ihrer zugrunde liegenden ethischen Positionen auf der Grundlage des präskriptiven Attributionsmodells analysiert (3.2).

\subsection{Identifikation von Rechtfertigungen}

Im Versuch, eine strittige Handlung zu legitimieren, geht es nicht um den Erweis von Wahrheit, sondern um die Begründung der Richtigkeit einer fraglichen Handlung. Rechtfertigungen basieren demnach auf bestehenden Werten, Normen und Konventionen und werden den normativen Argumentationsformen zugeordnet. Im Gegensatz zur traditionellen Logik folgen Alltagsargumentationen, wie wir sie in der Politik vorfinden, keinem strengen Schema von Prämissen und resultierenden Schlussfolgerungen. Zur Identifikation politischer Rechtfertigungen eignet sich daher das Klassifikationsmodell von Kienpointner, das sechs elementare Alltagsargumentationsmuster unterscheidet, die hier auf den politischen Kontext bezogen vorgestellt werden. Die Argumentationsformen sind nicht immer trennscharf voneinander abgrenzbar. Sie dienen jedoch als gute Selektionsgrundlage, um die Bandbreite der Erscheinungsformen zu berücksichtigen, die öffentliche Rechtfertigungen aufweisen können.

Einordnungsschemata liegen dann vor, wenn die strittige Handlung bereits durch die Einordnung der Situation oder die Einstufung des Gegners als begründet dargestellt wird. So ist aus der Analyse von Konfliktdynamiken bekannt, dass die Einordnung der Situation bereits Auswirkungen auf den späteren Umgang mit dem Konflikt hat. In einer Situation, die als >Herausforderung ‘ gesehen wird, scheinen andere Handlungen gerechtfertigt als in einem Konflikt, der als >Krieg`eingestuft wird: »Vor zwei Wochen wurde Amerika mit einem kriegerischen Akt der Krieg erklärt.« Oder: »The creation of Israel is a crime which must be erased. ${ }^{13}$ Neben der Situation wird auch der Gegner auf bestimmte, meist ausschließlich negative Weise kategorisiert. Gelegentlich wird er bereits bestehenden negativen Konzepten subsumiert, deren Bekämpfung als legitim angesehen wird. Die >Achse des Bösen < und der >Zionismus sind diesbezüglich prominente Beispiele unserer Zeit.

Vergleichsschemata. Schlussfolgerungen, die aus vergleichenden Argumentationen hervorgehen, können entweder eine Verschiedenheit oder Gleichheit betonen. Ein häufiger Typus von Vergleichskonklusionen ist die Betonung der Überlegenheit eigener Werte im Vergleich zu den Motiven des Gegners. Bei der Gleichsetzung hingegen wird auf ähnliche Konfliktsituationen verwiesen, in denen ein analoges Vorgehen von der eigenen oder gegnerischen Seite als gerechtfertigt angesehen wurde: »If Sharon is a man of peace in the eyes of Bush, then we are also men of peace.«

Gegensatzschemata basieren auf binärer Logik und antagonistischen Vorstellungen. Ein Gegensatzschema liegt beispielsweise vor, wenn neben der Idealisierung der eigenen Ziele die Berechtigung der gegnerischen Handlung vollständig geleug-

13 W. Kempf (2000). Gewaltursachen und Gewaltdynamiken. In ÖSFK (Hrsg.). Konflikt und Gewalt. Ursachen - Entwicklungstendenzen - Perspektiven (S. 44-65). Münster: Agenda. 
net wird: »The believers fight for Allah's cause, while those who reject faith fight for an unjust cause.« In einigen Fällen wird der Konflikt auf den Kampf zwischen »Gut und Böse« reduziert: »Die Menschen, die diese Anschläge gegen Amerika verübt haben und möglicherweise weitere Anschläge planen, sind böse Menschen. (...) Das Böse ist alles, woran sie denken können. Und als eine Nation guter Menschen werden wir auf sie Jagd machen.«14

In den Kausalschemata wird eigenes (Wohl-)Verhalten von dem des Gegners abhängig gemacht. Dies ist Ausdruck der Konfliktspirale, die beschreibt, dass die jeweiligen Parteien meinen, lediglich auf die Handlungen des Gegners zu reagieren: »Why are we fighting and opposing you? The answer is very simple: Because you attacked us and continue to attack us. $\ll^{15}$

Analogieargumentationen liegen dann vor, wenn die Situation anhand von Bildern beschrieben wird: »The situation was like a crocodile meeting a helpless child, powerless except for his screams.« Ist eine Bewertung der Situation eindeutig erkennbar, kann eine Analogie als Teil eines normativen Schemas gelten, wenn sie eine Begründung für das jetzige Handeln beinhaltet (hier: das als brutal empfundene Vorgehen der internationalen Truppen im Libanon).

Autoritätsargumentationen sind Rechtfertigungsformen, in denen sich der Redner auf relevante Persönlichkeiten bezieht, die zu einem Konflikt in bestimmter Weise Stellung bezogen haben. Als Autorität können hier sowohl eine Person als auch eine göttliche Macht gesehen werden: »So we are continuing this policy in bleeding America to the point of bankruptcy. Allah willing, and nothing is too great for Allah.«

Um eine objektive Identifikation von Rechtfertigungen zu gewährleisten, sollte die Beurteilung mittels eines Ratingprozesses von mindestens zwei Ratern vollzogen werden. Es sollten anschließend lediglich Aussagen aufgenommen werden, die von jeweils zwei Beurteilern unabhängig voneinander als Rechtfertigung eigenen Handelns identifiziert wurden.

\subsection{Die Analyse ethischer Grundpositionen}

\subsubsection{Das Präskriptive Attributionsmodell}

In einem zweiten Ratingprozess werden die selektierten Rechtfertigungen hinsichtlich der ihnen zugrunde liegenden ethischen Positionen analysiert. Grundlage der empirischen Untersuchung ist das präskriptive Attributionsmodell. ${ }^{16}$ Es basiert auf der allgemein anerkannten Unterscheidung zwischen Pflicht- und Zweckethiken. Während letztere die moralische Richtigkeit von Handlungen ausschließlich in Hinblick auf die Konsequenzen beurteilen, werden in den Pflichtethiken

14 US-Präsident George W. Bush vor den Mitarbeitern des FBI am 25. September 2001. Quelle: http://amerikadienst.usembassy.de/us-botschaft-cgi/ad-detailad.cgi?lfdnr=\%201335 (abgerufen am 25.2.2007).

15 Bin Laden, O. (2002). To the Americans. October 6, 2002. In: B. Lawrence (Hrsg.) (2005), Messages to the World. The Statements of Osama bin Laden (S. 162). London: Verso.

16 Ebenda, S. 165
Handlungen aufgrund der ihnen inhärenten Aspekte bewertet, die ungeachtet der Konsequenzen an sich als richtig oder falsch gelten. »Insofern ergeben die beiden Typen von Ethik auf die Frage, was für sie eine moralisch ideale Welt ausmacht, radikal verschiedene Antworten. Für den (...) Konsequenzialisten ist die moralisch ideale Welt dadurch bestimmt, dass alle von ihm anerkannten Güter maximal realisiert sind, gleichgültig, ob dies durch moralisch wertvolles Handeln (...) oder aufgrund natürlicher Faktoren geschieht. Für den strengen Deontologen kann eine Welt schon dann als ideal gelten, wenn in ihr alle deontologischen moralischen Gebote und Verbote maximal befolgt werden. ${ }^{17}$

Neben der Unterscheidung zwischen Zweck- und Pflichtethiken differenziert das präskriptive Attributionsmodell zwischen drei unterschiedlichen Betrachtungsebenen - dem individuellen, gruppenspezifischen und allgemeinen Beurteilungsbezug. Während Argumentationen auf der allgemeinen Beurteilungsebene Bezug auf die Menschheit an sich nehmen, ist die gruppenspezifische Betrachtungsebene in der Argumentation auf eine Gruppe begrenzt - etwa auf eine bestimmte Nation oder eine Religionsgemeinschaft. Gruppenspezifische Rechtfertigungen zeigen daher einen deutlich partikularistischen Charakter und grenzen sich somit deutlich von universellen Begründungen moralischen Handelns ab. Die individuelle Ebene fokussiert hingegen auf Aspekte moralischen Handelns des Individuums. Aus der Kombination der Dimensionen und ihrer Ausprägungen entsteht ein 2x3-Felder-Schema (Tabelle 1), aus dem sechs verschiedene Ethiken hervorgehen: Deontologie, Partikuläre Deontologie, Utilitarismus, Partikulärer Utilitarismus, Intuitionismus und Hedonismus. Die einzelnen Ethiken werden im Folgenden in der eben genannte Reihenfolge beschrieben.

Tabelle 1: Das Präskriptive Attributionsmodell

\begin{tabular}{|l|l|l|}
\cline { 2 - 3 } \multicolumn{1}{c|}{} & Zweck-Ethiken & Pflicht-Ethiken \\
\hline $\begin{array}{l}\text { Individueller } \\
\text { Beurtei- } \\
\text { lungsbezug }\end{array}$ & $\begin{array}{l}\text { Hedonismus } \\
\text { Der Redner betont, dass } \\
\text { im Interesse und zum } \\
\text { Wohle des Einzelnen } \\
\text { gehandelt werden muss. }\end{array}$ & $\begin{array}{l}\text { Intuitionismus } \\
\text { Der Redner betont, davon } \\
\text { überzeugt zu sein, dass die } \\
\text { Handlung richtig und } \\
\text { angemessen ist. }\end{array}$ \\
\hline $\begin{array}{l}\text { Gruppen- } \\
\text { spezifischer } \\
\text { Beurtei- } \\
\text { lungsbezug }\end{array}$ & $\begin{array}{l}\text { Partikulärer Utilitarismus } \\
\text { Der Redner verweist auf } \\
\text { die positiven Konse- } \\
\text { quenzen der Handlung } \\
\text { für eine Gruppe. }\end{array}$ & $\begin{array}{l}\text { Partikuläre Deontologie } \\
\text { Der Gegner verweist auf } \\
\text { gruppenspezifische Rechte } \\
\text { und Pflichten, nach denen } \\
\text { gehandelt werden muss. }\end{array}$ \\
\hline $\begin{array}{l}\text { Allgemeiner } \\
\text { Beurteilungs- } \\
\text { bezug }\end{array}$ & $\begin{array}{l}\text { Utilitarismus } \\
\text { Der Redner verweist auf } \\
\text { den großen Nutzen der } \\
\text { Handlung für die } \\
\text { Allgemeinheit. }\end{array}$ & $\begin{array}{l}\text { Deontologie } \\
\text { Der Redner spricht von all- } \\
\text { gemeingültigen Prinzipien, } \\
\text { nach denen grundsätzlich } \\
\text { gehandelt werden muss. }\end{array}$ \\
\hline
\end{tabular}

\section{Deontologie}

Deontologische Urteile (griechisch déov - Pflicht, Schuldigkeit, das Nötige) bewerten nach Broad (1887-1971) die moralische Richtigkeit oder Falschheit von Handlungen auf der Grundla-

17 Ebenda, S. 161. 
ge bestimmter Merkmale der Handlungen selbst. Ausschlaggebend für die positive Bewertung ist, dass Handlungen mit guter, prinzipientreuer Absicht vollzogen werden. In deontologischen Begründungen wird daher betont, entsprechend allgemeingültiger Gebote, Normen oder Konventionen zu handeln, die verbindliche Pflichten, aber auch Rechte darstellen. »Ob wir unsere Feinde zur Rechenschaft ziehen oder unsere Feinde ihrer gerechten Bestrafung zuführen, der Gerechtigkeit wird Genüge getan werden.« 18

Partikuläre Deontologie. Bei der Partikulären Deontologie gilt die Besonderheit, dass eine Handlung auf der Grundlage von gruppenspezifischen Normen beurteilt wird. Für jedes Gruppenmitglied gibt es klare Regeln, die sich von Verhaltensregeln für Außenstehende unterscheiden. Die gruppenspezifischen Handlungsnormen können sowohl auf bestimmten Verpflichtungen als auch auf besonderen Rechten basieren: »Die Anweisung, Amerikaner und ihre Verbündeten - Zivilisten und Militärs - zu töten, ist eine individuelle Pflicht für jeden Muslimen, der dies in jedem Land tun kann, in dem dies möglich ist. ${ }^{19}$

Utilitarismus. Die Begründung des Utilitarismus (lat. utilis - nützlich) geht auf Jeremy Bentham (1748-1832) zurück. Er charakterisiert die am weitesten ausgearbeitete und meist verbreitete Variante der konsequenzialistischen Ethiken. Bei der Bewertung der Handlungsfolgen muss der Nutzen aller im Vordergrund stehen - der Akteur darf sich und die ihm Nahestehenden nicht privilegieren. Charakteristisch für den Utilitarismus sind eine ausgeprägte Zukunftsorientierung und das Denken in langfristigen Entwicklungstendenzen: »Damit verteidigen wir nicht nur unsere Freiheit, sondern auch die Freiheit der Menschen überall auf der Welt. $\ll^{20}$

Partikulärer Utilitarismus. Im Gegensatz zum allgemeinen Utilitarismus gilt hier die Einschränkung, dass die Handlung nicht universell für alle Betroffenen von größtem Nutzen sein muss, sondern dass die Handlung dann als gut beurteilt wird, wenn sie für eine bestimmte Gruppe, wie die eigene Nation, Vorteile birgt: »Wir werden diese entscheidende Schlacht dazu benutzen, uns auf den Stand der modernen Wissenschaft und Organisation emporzuarbeiten. $\ll^{21}$

Intuitionismus. Grundlage, auf der intuitionistische Urteile gefällt werden, ist die individuelle Einsicht. Diese umfasst Vormeinungen und andere Arten vortheoretischer moralischer Überzeugungen, aufgrund derer die Richtigkeit einer Handlung eingeschätzt wird. Ein wichtiges Merkmal von Intuitionismus ist zudem, dass die Urteile unmittelbar gefasst werden und nicht auf logischer Ableitung beruhen: »In those critical moments, I was overwhelmed by ideas that are hard to describe, but they awakened a powerful impulse to reject injustice and gave birth to a firm resolve to punish the oppressors. «22

18 George W. Bush in der Ankündigung des »Kriegs gegen den Terror « am 20.9.2001. Quelle: http://usa.usembassy.de/etexts/docs/ga1-092001d.htm (abgerufen am 25.2.2007).

19 Bin Laden, zitiert nach >Der Spiegek vom 24.8.1998, S.120.

20 Bush zu den Militärschlägen in Afghanistan am 7. Oktober 2001. Quelle: http://amerikadienst.usembassy.de/us- botschaft-cgi/ad-detailad. cgi?lfdnr=1504 (abgerufen am 25.2.2007).

21 Aflak, M. (1978). Arabische Sozialistische Ba'th Partei. Auszüge aus Reden, Erklärungen und Interviews (S. 6). Varese: Valle Olona.

22 Bin Laden am 29.10.2004, vgl. Fußnote 20.
Hedonismus. Die Grundlage, auf der hedonistische Rechtfertigungen von Handlungen beruhen, sind Konsequenzen, die sich auf das physische und psychische Wohlergehen eines Individuums beziehen: »Should a man be blamed for protecting

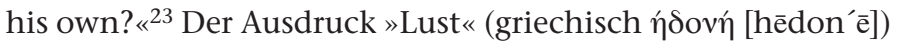
ist dabei leicht irreführend, da er nahe legt, dass es dem Hedonisten vorrangig um körperliche oder sinnliche Lust geht. Dies ist jedoch keineswegs der Fall. »Intrinsisch wertvoll ist für den Hedonisten Wohlbefinden, ein positiv getönter Empfindungszustand, gleichgültig, ob sich dieser aus körperlichen oder geistigen Quellen speist. ${ }^{24}$

\subsubsection{Die Unterscheidung direkter und indirekter Rechtfertigungen}

Im Verlauf von Analysen öffentlicher Rechtfertigungen zeigte sich, dass die sechs ethischen Grundpositionen sowohl in direkter als auch in indirekter Art vorliegen können. Charakteristisch für die direkte Rechtfertigungsform ist das Aufführen von Gründen und Prinzipien, die der Verletzung moralischer Normen durch das eigene Handeln entgegengestellt werden. Eine indirekte Rechtfertigung besteht hingegen dann, wenn Normen- und Werteverletzungen des Gegners betont werden, um das eigene Handeln zu rechtfertigen: »Wir haben heute mit dem Kommando Vincenzo Spano die Botschaft der USA in Bonn beschossen, weil die USA im Vernichtungskrieg gegen das irakische Volk von Anfang an die Führungsrolle übernommen haben. «25 Das eigene Handeln wird somit als Kompensation der gegnerischen Werteverletzung dargestellt. In Tabelle 2 finden sich Prinzipien und Beispiele indirekter Rechtfertigungsformen.

\subsubsection{Der Codierprozess}

Um eine objektive Auswertung zu gewährleisten, wird das relevante Material von mehreren Ratern unabhängig voneinander analysiert. Dafür gilt es zunächst, adäquate Kontext- und Codiereinheiten festzulegen. Während Letztere bestimmt, welche Texteinheit (Wort, Phrase, Satz, Argumentationsstrang, Absatz) als Bedeutungstragende Einheit kategorisiert werden soll, ist die Kontexteinheit die Einheit, in der »der Codierer nach Begründungen für begründungsbedürftige Codiereinheiten sucht « ${ }^{26}$. In den hier berichteten Analysen von politischen Rechtfertigungen wurde der Satz als Codiereinheit gewählt. Neben der leichten und objektiven Bestimmbarkeit hat dies den Vorteil, dass Prinzipien, die über mehrere Sätze betont werden und daher in den öffentlichen Reden viel Raum einnehmen, ebenso in der Codierung eine besondere Gewichtung erhalten. Als Kontexteinheit wurde der Themenabschnitt gewählt, der in einigen Fällen weitere Hinweise für die Bestimmung ethischer

23 Ebenda

24 Birnbacher, S. 254.

25 RAF in einer Erklärung vom 13. Februar 1991. In M. Hoffmann (1997). Rote Armee Fraktion. Texte und Materialien zur Geschichte der RAF . Berlin: IDVerlag.

26 P. Mayring (2003). Qualitative Inhaltsanalyse. Grundlagen und Techniken (8. Aufl.), (S. 53). Weinheim: Beltz-PVU. 
Tabelle 2: Indirekte Rechtfertigungen eigenen Handelns

\begin{tabular}{|c|c|}
\hline Verstoß des Gegners gegen Zweck-Ethiken & Verstoß des Gegners gegen Pflicht-Ethiken \\
\hline Indirekter Hedonismus (H-) & Indirekter Intuitionismus (I-) \\
\hline $\begin{array}{l}\text { Der Redner verurteilt Aktionen des Gegners, die dem Wohl- } \\
\text { ergehen und der Unversehrtheit Einzelner entgegenstehen: } \\
\text { »Buddenberg, das Schwein, hat Grashof zu einem Zeitpunkt } \\
\text { vom Krankenhaus in die Zelle verlegen lassen, als der Trans- } \\
\text { port und die Infektionsgefahr im Gefängnis noch lebensge- } \\
\text { fährlich für ihn waren. «1 }\end{array}$ & $\begin{array}{l}\text { Der Redner verurteilt den fehlenden 'gesunden Menschen- } \\
\text { verstand' des Gegners. } \\
\text { »Those who condemn these operations [9/11] have viewed } \\
\text { the event in isolation and have failed to connect it to previ- } \\
\text { ous events or to the reasons behind it. Their view is blinkered } \\
\text { and lacks either a legitimate or a rational basis. }{ }^{2}\end{array}$ \\
\hline Indirekt-partikulärer Utilitarismus (PU-) & Indirekt-partikuläre Deontologie (PD-) \\
\hline $\begin{array}{l}\text { Der Redner verweist auf die (potenzielle) Gefahr der gegn- } \\
\text { erischen Handlung für eine bestimmte Gruppe: } \\
\text { »We're concerned that Iraq is exploring ways of using these } \\
\text { UAVS for missions targeting the United States. } \aleph^{3}\end{array}$ & $\begin{array}{l}\text { Der Redner betont, dass die speziellen Pflichten des Gegners } \\
\text { nicht eingehalten wurden: »Wir werden sooft und solange } \\
\text { Sprengstoffanschläge gegen Richter und Staatsanwälte } \\
\text { durchführen, bis sie aufgehört haben, gegen die politischen } \\
\text { Gefangenen Rechtsbrüche zu begehen. «1 }\end{array}$ \\
\hline Indirekter Utilitarismus (U-) & Indirekte Deontologie (D-) \\
\hline $\begin{array}{l}\text { Der Redner betont die (potenzielle) Gefahr des gegnerischen } \\
\text { Handelns für die Allgemeinheit: } \\
\text { »This enemy attacked not just our people, but all freedom-lo- } \\
\text { ving people everywhere in the world. }{ }^{4}\end{array}$ & $\begin{array}{l}\text { Der Redner betont, dass der Gegner nicht entsprechend } \\
\text { allgemein anerkannter Normen und Werte handelt: »And } \\
\text { by the will of God Almighty, we will soon see the fall of the } \\
\text { unbelievers' states, at whose forefront is America, the tyrant, } \\
\text { which has destroyed all human values. } «^{5}\end{array}$ \\
\hline
\end{tabular}

1 RAF in einer Erklärung vom 20.5.1972. In: M. Hoffmann (1997). S. 146

2 Bin Laden am 26.12.2001. In: B. Lawrence (2005). S. 149.

3 G. W. Bush am 7.10.2002. Quelle: http://amerikadienst.usembassy.de/us-botschaft-cgi/ad-detailad.cgi?lfdnr=1504

4 G. W. Bush am 12.9.2001. Quelle: http://www.whitehouse.gov/news/releases/2002/10/20021007-8.html

5 Bin Laden am 25.8.2002. In: B. Lawrence (2005). S. 159.

Prinzipien in einem Satz erbringen kann. Bevor das relevante Material codiert wird, muss die Bestimmung ethischer Grundpositionen von Ratern anhand von Übungsmaterial trainiert werden, bis sich eine zufriedenstellende Beurteilungsübereinstimmung abzeichnet. Diese sollte durch die Berechnung von Übereinstimmungsmaßen für nominalskalierte Daten (Cohen's kappa) kontrolliert werden. ${ }^{27}$

\subsubsection{Der Auswertungsprozess}

Die Argumentationsmuster der untersuchten Gruppen können zunächst deskriptiv dargestellt werden. Unterschiede zwischen den Gruppen und vorher aufgestellte Hypothesen sollten inferenzstatistisch überprüft werden. Da es sich um nominalskalierte Daten handelt, eignen sich dazu nonparametrische Verfahren wie einfache $\mathrm{Chi}^{2}$-Tests oder Konfigurationsfrequenzanalysen. ${ }^{28} \mathrm{Um}$ die Größe der Unterschiede näher bestimmen zu können, sollten zudem Effektstärken berechnet werden. ${ }^{29}$

27 J. Bortz (2005). Statistik für Human- und Sozialwissenschaftler (6. Aufl.). Heidelberg: Springer.

28 J. Krauth (1993). Einführung in die Konfigurationsfrequenzanalyse (KFA). Ein multivariates nichtparametrisches Verfahren zum Nachweis und zur Interpretation von Typen und Syndromen. Weinheim; Basel: Beltz-PVU.

29 J. Cohen (1977). Statistical power analysis for the behavioral sciences. New York, NY: Acad. Press.

\section{Beispielhafte Ergebnisse}

In den bisherigen Analysen von ethischen Rechtfertigungen politisch motivierter Akte der Gewalt wurden Krieg führende Parteien und terroristische Organisationen aus westlichen und arabischen Ländern untersucht. Exemplarisch wurde dazu veröffentlichtes Material von vier Gruppen analysiert: (1) Erklärungen der US-amerikanischen Regierung zu den Militärschlägen gegen Afghanistan (2001- ) und zum IrakKrieg (2003- ) (2) Erklärungen der Roten Armee Fraktion zu Anschlägen in Deutschland zwischen 1972 und 1984 (3) Stellungnahmen des ehemaligen irakischen Präsidenten zum Iran-Irak-Krieg (1980-1988) und (4) ausgestrahlte Reden von führenden Al-Qaida-Mitgliedern zu terroristischen Akten zwischen 2001 und 2004. Insgesamt wurden 1253 Rechtfertigungen analysiert (NUSA $=479, \mathrm{NRAF}=125$, NIrak $=217$ und NAl-Qaida $=432$ ).

Von bedeutender Relevanz scheint im Kontext politischer Gewalt die indirekte Rechtfertigungsweise zu sein, die in den untersuchten Argumentationen weit häufiger auftritt $(60,15 \%)$ als Rechtfertigungsformen direkter Natur (39,85\%). Insbesondere die RAF (68,6\%) und der Irak $(69,2 \%)$ rechtfertigen ihre Handlungen durch gegnerische Verstöße gegen ethische Prinzipien, während die USA das feindliche Fehlverhalten zu 43,0\% als Grundlage ihres Handelns 
Abbildung 1: Rechtfertigungsstrukturen der untersuchten Gruppen

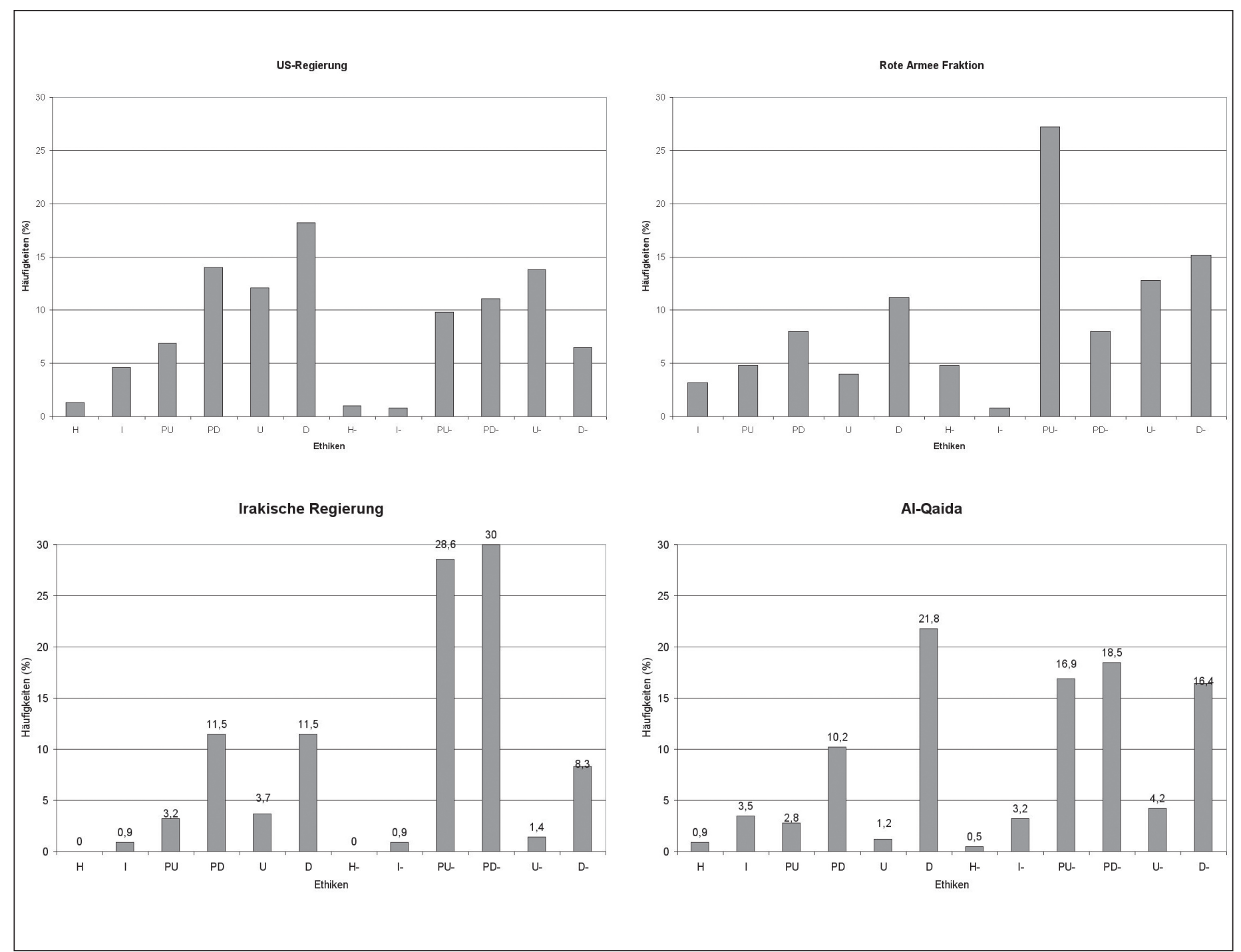

heranzieht und vornehmlich die Verfolgung eigener Prinzipien in den Vordergrund stellt.

Für die untersuchten Gruppen ergeben sich distinkte Rechtfertigungsmuster (Abb. 1). In den öffentlichen Erklärungen der US-amerikanischen Regierung findet sich eine relativ breite Rechtfertigungsstruktur, in der jede der zwölf ethischen Argumentationsformen in die Rechtfertigung des Irak-Krieges mit einbezogen wird. Inferenzstatistische Untersuchungen zeigen zudem, dass die US-amerikanische Regierung in ihren Ausführungen utilitaristische Aspekte (U) deutlich häufiger betont als alle anderen Gruppen. Sie unterstreicht damit, dass ihre Handlungen die bestmöglichen Konsequenzen für die Mehrheit der Menschen verkörpern.

Die Betonung der aktuellen oder potenziellen Gefahr des gegnerischen Handelns für die Menschheit (U-) scheint sowohl für die US-Regierung als auch die RAF typisch zu sein. Als
Hauptargumentationsform der Roten Armee Fraktion stellt sich jedoch der negativ-partikuläre Utilitarismus (PU-) heraus, mit dem auf die negativen Konsequenzen der gegnerischen Handlung für ein bestimmtes Volk verwiesen wird - wie z.B. für die nordvietnamesische Bevölkerung.

Zentrale Rechtfertigungsformen des Irak bezüglich des IranIrak-Krieges (1980-1988) sind die beiden negativ-partikulären Argumentationsformen der partikulären Deontologie und des partikulären Utilitarismus. Diese stellen die gegnerischen Verstöße hinsichtlich spezifischer Verpflichtungen (PD-) und den damit verbundenen Auswirkungen auf die eigene Gruppe (PU-) in den Vordergrund. Dass dies die Hauptargumentationsformen der irakischen Regierung darstellen, verwundert nicht, wenn man bedenkt, dass die Nicht-Beachtung des Algerien-Abkommens von 1975 einer der Hauptstreitpunkte in der Iran-Irak-Krise war. 
In den untersuchten Al-Qaida Reden stehen die Beachtung und Nicht-Beachtung allgemeiner und gruppenspezifischer Pflichtenethiken im Vordergrund (D, D-, PD, PD-). Innerhalb des konsequenzialistischen Spektrums scheint lediglich der negativ-partikuläre Utilitarismus (PU-) eine tragende Bedeutung zu haben, mit dem die negativen Auswirkungen der gegnerischen Handlung für eine bestimmte Gruppe - hier meist die muslimische Bevölkerung - betont werden.

Inferenzstatistische Vergleiche zeigen in den Argumentationsformen sowohl Gemeinsamkeiten als auch Diskrepanzen zwischen den Gruppen. Die meisten Unterschiede stellen sich zwischen den Argumentationen von Al-Qaida und der USamerikanischen Regierung heraus. Während die US-Regierung in ihren Reden betont, mit den Akten der Gewalt das maximale Wohl für die Allgemeinheit erreichen zu wollen $\left(\mathrm{U}, \mathrm{h}^{30}=.538\right)$ und die potenzielle Gefahr des Gegners von der Allgemeinheit abzuwenden ( $\mathrm{U}-, \mathrm{h}=.344)$, betonen Al-Qaida-Mitglieder im Gegensatz dazu die Verletzung von Pflichten des Gegners (PD-, $\mathrm{h}=.22$ ) und die resultierenden negativen Auswirkungen für die eigene Bevölkerung (PU-, $h=.20$ ). Die positiven Konsequenzen der jeweiligen Operation für eine bestimmt Gruppe werden hingegen von der US-Regierung unterstrichen (PU, $\mathrm{h}=$ .226). Die deontologische Rechtfertigungsform, in der eigene Handlungen auf Normen und Werte bezogen werden, die als allgemeingültig dargestellt werden, erweist sich als dominante Argumentationsweise beider Gruppen. Während beide es als ihre Pflicht betrachten, gegen den anderen vorzugehen, suggeriert jedoch die US-amerikanische Regierung, dies als Weltmacht im Interesse der Menschheit zu tun, wohingegen Al-Qaida argumentiert, aufgrund des verursachten Leids der muslimischen Bevölkerung gegen die unmoralischen Akte des Westens (D-, $\mathrm{h}=.34$ ) anzugehen. Zwischen der US-Regierung und der RAF, der RAF und Al-Qaida und der RAF und dem Irak bestehen weit weniger Unterschiede. Mittlere Unterschiede befinden sich in den Argumentationen des Irak und Al-Qaidas und zwischen der US-Regierung und dem Irak. ${ }^{31}$

\section{Diskussion}

Methodisch zeigt sich, dass die hier vorgestellten Modelle zur Identifikation und Analyse ethischer Rechtfertigungen einen inhaltsanalytischen Rahmen bilden, um Unterschiede in politischen Argumentationen auf der Werteebene abzubilden und auf zentrale Aspekte zurückzuführen. Das Klassifikationsmodell alltäglicher Argumentationsstrukturen von Kienpointner eignet sich trotz mangelnder Trennschärfe dazu, unterschiedlichste Formen ethischer Rechtfertigungen zu identifizieren. Als besonders bereichernd in der Analyse kulturell verschiedener Materialen erweist sich dabei der Einbezug von Analogieargumentationen, die in nicht-westlichen Texten eine

30 h steht für die Effektstärke prozentualer Differenzen. Cohen (1977, S. 184 f.) unterscheidet zwischen kleinen Effekten mit $\mathrm{h}=.20$, mittleren Effektgrößen mit $\mathrm{h}=.50$ und großen Effekten mit $\mathrm{h}=.80$.

31 Ergebnisse bezüglich weiterer Unterschiede zwischen den Gruppen sowie der kulturellen und gewaltabhängigen Besonderheiten (Witte \& Halverscheid, 2006) können hier nicht berichtet werden, sind aber unter http://www.unihamburg.de/fachbereiche-einrichtungen/fb16/absozpsy/HAFOS-70.pdf zu beziehen. bedeutende Rolle spielen. Die Anwendung des präskriptiven Attributionsmodells überzeugt zunächst darin, dass alle identifizierten Rechtfertigungen durch das Modell klassifiziert werden konnten. Pro Codiereinheit mussten jedoch Mehrfachcodierungen zugelassen werden, da einige Sätze mehr als ein ethisches Prinzip beinhalteten. Darüber hinaus ist zu betonen, dass die hier untersuchten Rechtfertigungen nicht unbedingt die moralische Überzeugung der Redner widerspiegeln. Sie reflektieren jedoch, wie die breite Öffentlichkeit von der Richtigkeit der Handlungen überzeugt werden soll. Es bleibt zu klären, ob die Begründungsmuster in Abhängigkeit von den Adressaten variieren oder konstant bleiben.

Deutlich unterrepräsentiert sind die vier ethischen Grundpositionen mit individuellem Beurteilungsbezug (H, H-, I und I-). Diese Ebene wurde in anderen Kontexten häufiger beobachtet, ${ }^{32}$ scheint jedoch im Rahmen der hier behandelten Thematik als weniger überzeugend zu gelten. Die direkte Form des Intuitionismus (I), mit der eigene Handlungen entsprechend der individuellen Einsicht als unausweichlich dargestellt werden, ist bei der Rechtfertigung von Krieg und Terror mit insgesamt 12,2\% die am häufigsten gefundene Rechtfertigungsform mit individuellem Beurteilungsbezug.

Inhaltlich erweisen sich im Kontext politischer Gewalt sowohl die Differenzierung zwischen gruppenspezifischen und allgemeinen Beurteilungsbezügen als auch die indirekten Rechtfertigungsformen als adäquat. So tragen die Ebenen in ihrer Kombination dazu bei, das bestehende Spannungsverhältnis bezüglich der Beurteilung eigener und fremder Werte konkret zu erfassen. Das Vorherrschen der indirekten Rechtfertigung reflektiert dabei die starke Neigung, das gegnerische Handeln zu verurteilen. In den bisherigen Untersuchungen weicht lediglich die US-amerikanische Regierung von diesem Muster etwas ab. Sie zeigt sich aufgrund der äußeren Bedrohung deutlich weniger beunruhigt als die anderen Gruppen, was zum Teil durch ihre überlegene Machtposition erklärt werden kann.

Trotz der herausgestellten Unterschiede zwischen den Gruppen muss festgehalten werden, dass alle zwölf ethischen Grundpositionen sowohl in der Rechtfertigung von Krieg und Terror als auch im arabischen und westlichen Raum festgestellt wurden. Diesbezüglich äußert Birnbacher: »Was zwischen den Zeiten und den Kulturen variiert, ist weniger der Bestand an Werten und Normen als vielmehr ihr relatives Gewicht und die ihnen jeweils zugeschriebenen Rangfolgen. Nicht die Listen von Werten und Normen unterscheiden sich, sondern die Plätze, die diese Werte und Normen auf den Listen einnehmen.«33 Dieser Aspekt wird allzu sehr vernachlässigt. Es gilt, hinter den extremen Äußerungen, die als radikal und bedrohlich wahrgenommen werden, übereinstimmende Handlungsprinzipien zu finden, die von beiden Seiten anerkannt werden können.

\footnotetext{
32 E. H. Witte \& J. Doll (1995), vgl. Fußnote 2; E. H. Witte \& C. Mölders (2006). Einkommenssteuergesetz: Begründung der vorhandenen Ausnahmetatbestände ethisch bedenklich. (Hamburger Forschungsbericht zur Sozialpsychologie Nr. 69). Hamburg: Universität Hamburg, Arbeitsbereich Sozialpsychologie.

33 Birnbacher (2003) S. 391
} 